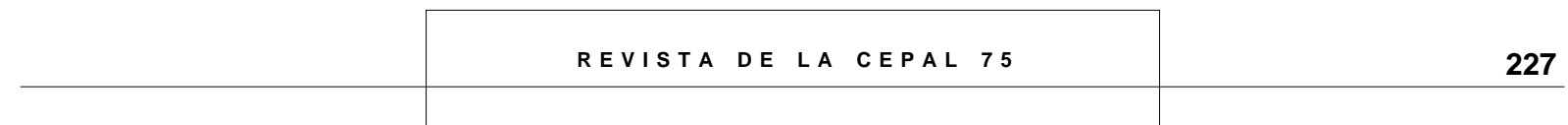

\title{
Impactos estáticos y dinámicos del MERCOSUR. El caso del sector farmacéutico
}

\author{
Marta Bekerman \\ Directora \\ bekerman@econ.uba.ar \\ En este artículo se analiza el impacto del Mercosur sobre el \\ Pablo Sirlin \\ sector farmacéutico. Entre las conclusiones surge que, como \\ Investigador \\ psirlin@sepyme.gov.ar \\ Centro de Estudios de la \\ consecuencia del proceso de integración, el sector sufrió fuer- \\ tes impactos de carácter tanto estático como dinámico. Desde \\ Estructura Económica (CENES), \\ Facultad de Ciencias \\ Económicas, \\ Universidad de \\ Buenos Aires \\ el punto de vista estático, puede apreciarse que la integración \\ ha dado lugar a un notable aumento del comercio intrazonal, \\ lo que revela la existencia de un importante proceso de crea- \\ ción de comercio en el Mercosur. Desde el punto de vista di- \\ námico, el aspecto saliente es el interés cada vez mayor mos- \\ trado por las empresas trasnacionales, a partir del proceso de \\ integración, por reposicionarse en los mercados de Argentina \\ y Brasil. Este proceso, junto con los cambios regulatorios en \\ materia de patentes medicinales, ha generado un fuerte cambio \\ de estrategia y posicionamiento de los laboratorios de origen \\ nacional.
}




\section{I}

\section{Introducción}

La evaluación de los beneficios de los procesos de integración, así como de sus costos, no sólo es una tarea difícil, sino que no existe consenso sobre la manera de llevarla adelante.

La mayor parte de los estudios económicos consideran que los efectos estáticos de creación o desvío de comercio son la variable fundamental para tal objetivo. Sin embargo, esos efectos dejan afuera los beneficios potenciales de carácter dinámico que pueden derivarse del proceso de integración, como el desarrollo de cambios tecnológicos, de procesos de aprendizaje, de especialización productiva y otros. Estos efectos dinámicos son los que ofrecen los argumentos más convincentes para defender los procesos de integración por sobre los de la apertura unilateral de la economía. Pero el problema se plantea a partir de las dificultades que existen para realizar su medición, ya que ésta no resulta del análisis de las tasas de crecimiento o del comportamiento de los flujos comerciales regionales.

Dichas dificultades plantean la necesidad de trabajar con dimensiones más desagregadas que permitan identificar procesos que no podrían ser detectados a nivel global. Para hacerlo, se procede a analizar las transformaciones sufridas en el interior de cada sector productivo, ${ }^{1}$ para determinar cómo fueron afectados los niveles y las formas de organización de la producción, las estrategias empresariales, los niveles de actualización tecnológica, la dinámica regional del sector, etc. Este tipo de análisis requiere aislar, en la medida de lo posible, los efectos de los procesos de integración de aquellos emanados de las demás transformaciones que están teniendo lugar en las economías de la zona (procesos de apertura, de privatizaciones y otros). Esto nos dará elementos para descubrir qué tipo de políticas nacionales o comunitarias podrían aplicarse en el futuro para hacer del Mercosur un dinamizador del desarrollo productivo.

Siguiendo el criterio metodológico descrito, en este artículo se analizará el impacto del Mercosur so-

\footnotetext{
${ }^{1}$ Es que cada sector se ve afectado en forma muy distinta, ya sea por la situación competitiva previa, por las posibilidades que existen de aprovechar demandas latentes de los restantes mercados, o por la forma particular en que inciden las asimetrías regulatorias entre países socios.
}

bre el sector farmacéutico. ${ }^{2}$ Se trata de un sector de gran interés para el análisis de los efectos de la integración regional, por su importancia estratégica, porque ha mostrado flujos de comercio intrazonales y extrazonales crecientes y por los profundos cambios que está experimentando tanto a nivel mundial como dentro de los países de la zona. En efecto, el costo y la forma de financiar el acceso de la población a los medicamentos es, y ha sido siempre, un fuerte motivo de preocupación de los gobiernos. Por otro lado, el nivel de innovación tecnológica y de eficiencia dinámica que alcance el sector puede ser un factor importante de su inserción en los mercados regionales y mundiales.

A modo de anticipo, cabe señalar que el sector, como consecuencia del proceso de integración, sufrió fuertes impactos de carácter tanto estático como dinámico. En primer término, la integración zonal ha dado lugar a un notable aumento del comercio intrazonal, puesto que las importaciones extrazonales también han aumentado y que los niveles de producción muestran crecimientos mucho menores, puede inferirse que existe un importante proceso de creación de comercio en el Mercosur. Los impactos dinámicos responden a diferentes causas, entre las cuales merece destacarse el creciente interés mostrado por las empresas trasnacionales, a partir del proceso de integración, por reposicionarse en los mercados de Argentina y Brasil (dadas las mayores posibilidades de expandir el comercio intrafirma).

El sector farmacéutico presenta un creciente grado de internacionalización, y está dominado a nivel mundial por un conjunto reducido de empresas, cuyo esfuerzo competitivo se basa en la permanente introducción de nuevos productos sobre la base de investigación científica y tecnológica, así como en fuertes gastos de comercialización y publicidad para los productos existentes. Dentro de la región son precisamente las

\footnotetext{
${ }^{2}$ El sector farmacéutico forma parte de una cadena productiva que se inicia con el descubrimiento de una molécula o principio activo y continúa con la producción industrial de fármacos. Estas dos etapas constituyen la industria "farmoquímica" y son parte de lo que generalmente se denomina "industria química fina". La industria farmacéutica propiamente dicha, que será analizada en este trabajo, engloba la producción de especialidades farmacéuticas o medicamentos y su comercialización.
} 
grandes firmas trasnacionales las que claramente dominan en el segmento de los medicamentos éticos patentados. ${ }^{3}$ Estas firmas actúan en forma muy dinámica en lo que se refiere tanto a la ubicación de sus plantas como a sus patrones de especialización nacional.

En el caso de los productos genéricos, la capacidad innovativa y los gastos de comercialización tienen un rol secundario; la competencia en precios es lo fundamental, y esto obliga a prestar una mayor atención a la tecnología de procesos y al aprovechamiento de economías de escala.

En la mayor parte de los países se encuentran, además de las trasnacionales, empresas de capital nacional que se dedican a la elaboración de especialidades farmacéuticas. Estas empresas utilizan como insumos fármacos genéricos a precios menores que los similares patentados, y prácticamente no realizan actividades de investigación, aunque sí tienen cierto nivel de gastos en desarrollo de productos. Compiten entre sí y con las filiales de las grandes empresas trasnacionales sobre la base de precios y de gastos en comercia- lización, siendo la marca y la imagen de la empresa un instrumento fundamental de competencia. En el caso de los productos éticos el esfuerzo de comercialización se dirige a la profesión médica, que es la que indica al paciente uno u otro medicamento. En los productos de venta libre la competencia se basa en la marca, consolidada a través de intensa publicidad a nivel masivo.

La propia naturaleza de los productos de esta industria, destinados a la atención de la salud pública, así como la importancia de las actividades de investigación científica y tecnológica hacen que se trate de un sector fuertemente regulado. ${ }^{4}$

Observaremos en primer lugar las asimetrías regulatorias que existen entre Argentina y Brasil (sección II) y el desempeño del sector en los distintos países del Mercosur (sección III), para finalmente evaluar los efectos estáticos y dinámicos del proceso de integración que es posible identificar a partir del análisis estadístico y de los estudios de campo realizados en Brasil, Uruguay y Argentina ${ }^{5}$ (sección IV).

\section{II}

\section{Asimetrías de política en el Mercosur}

Las asimetrías de política en el Mercosur se manifiestan en dos campos: por un lado, en las políticas globales de promoción a la inversión, la producción, la innovación y las exportaciones, y por otro, en las diferencias en el marco regulatorio que afecta específicamente al sector farmacéutico.

Durante la fase de industrialización sustitutiva de las importaciones los países del Mercosur, especialmente Argentina y Brasil, desarrollaron un vasto conjunto de instrumentos de promoción que combinaron altas tasas de protección comercial con regímenes especiales de incentivo a la inversión y, posteriormente, con instru-

\footnotetext{
3 Dentro de los medicamentos de uso humano se distinguen los éticos y no éticos. El primer grupo comprende a todos aquellos que se venden exclusivamente a través de receta médica, y pueden ser clasificados en genéricos y patentados. Los genéricos son aquéllos cuya patente ya expiró y pueden ser elaborados libremente. Los patentados, en cambio, en el caso de países que aceptan las reglas en la materia, sólo pueden ser fabricados por quienes poseen la patente o bajo licencia otorgada por los dueños de la patente original, por lo que sus precios son generalmente más elevados. Los medicamentos no éticos son aquellos que pueden ser adquiridos sin necesidad de receta médica.
}

mentos de estímulo a las exportaciones. Hacia fines del decenio de 1980 iniciaron un proceso de reformas orientadas a la liberalización económica y a desmontar el aparato de promoción y regulación del Estado, a las que no resultó ajeno el sector farmacéutico.

Sin embargo, en el caso brasileño todavía permanecen vigentes (y, en muchos casos, superpuestos) distintos incentivos a la inversión del gobierno federal y los gobiernos estaduales. Estos últimos disfrutan de potestades fiscales más amplias que las correspondientes a

\footnotetext{
${ }^{4}$ Las disposiciones sobre patentes establecidas en 1994 en los acuerdos sobre aspectos comerciales de los derechos de propiedad intelectual (TRIPS) del Acuerdo General de Aranceles Aduaneros y Comercio (GATT) obligan a los países, desde su entrada en vigencia, a brindar protección mediante patentes a los productos farmacéuticos y los procesos respectivos. Si bien a los países en desarrollo se les concedió un plazo de cinco años, estos acuerdos están teniendo un profundo impacto sobre aquellos países que no reconocían patentes y que habían logrado desarrollar una industria basada en esfuerzos de desarrollo imitativos por parte de empresas locales (como en India y Argentina).

${ }^{5}$ Los estudios de campo consistieron en la realización de una entrevista normalizada a alrededor de quince firmas de cada uno de los países y a las principales cámaras empresarias del sector.
} 
las provincias argentinas, por lo que tienen un importante margen para otorgar incentivos fiscales bajo la forma de reducción o financiamiento del impuesto a la circulación de mercaderías y servicios (ICMS) para atraer nuevas inversiones. También son usuales los incentivos estaduales a la capacitación de mano de obra y al desarrollo de infraestructura.

En el caso de Uruguay, las reformas estructurales han sido más graduales (prácticamente no existen procesos de privatización de empresas públicas), aunque durante la década de 1990 se profundizó el proceso de apertura unilateral del comercio (Laens, Lorenzo y Osimani, 1993; Torrello y Noya, 1992).

Por otro lado, desde inicios del decenio se nota la aparición de nuevos instrumentos de política dirigidos a fomentar el desarrollo tecnológico y la reestructuración empresarial. Ejemplos de ello son la creación del Fondo Tecnológico Argentino (FONTAR), orientado a fomentar la innovación tecnológica, y también una serie de incentivos a las exportaciones, como la admisión temporaria y los reintegros a la exportación, que han sido utilizados por la mayoría de los laboratorios argentinos entrevistados.

Dentro de las tendencias generales a racionalizar y reducir la intervención pública en materia de política industrial hubo en cada uno de los países del Mercosur sustanciales modificaciones en las políticas regulatorias del sector farmacéutico, el cual estuvo sujeto hasta fines del decenio de 1980 a fuertes restricciones vinculadas con los mecanismos de formación de precios y con el abastecimiento externo. En el decenio de 1990 tanto en Argentina como en Brasil se adoptó un conjunto de medidas desregulatorias que se iniciaron con la eliminación de los controles sobre los precios máximos, aunque subsisten importantes diferencias regulatorias entre ambos países que se manifiestan en dos esferas: la referente al registro de medicamentos y la vinculada a la ley de patentes.

Con respecto al registro de medicamentos, Argentina procedió a su agilización para facilitar la importación de fármacos como forma de incentivar la competencia con la producción local. Con ese propósito creó en 1992 el registro especial de medicamentos autorizados. ${ }^{6}$ En Brasil, en cambio, el mecanismo de registro de los medicamentos es mucho más restricti-

\footnotetext{
${ }^{6}$ Se estableció entonces el registro automático para todas las especialidades medicinales que ya circulaban en el país, y para aquellas autorizadas en los mercados internos de un conjunto de países considerados de "alta vigilancia sanitaria", cuya lista figura en el anexo I del decreto pertinente. Por otro lado, se determinó un plazo máximo de 120 días para decidir la autorización y registro de productos similares a otros ya inscritos en el país, ya sea para su elaboración interna o para su importación desde países incluidos en la lista del anexo II.
}

vo, ya que requiere la presentación de muestras para análisis y una serie de experiencias que sean juzgadas necesarias por el órgano sanitario. Estos requisitos ponen un serio límite a las importaciones brasileñas de medicamentos desde los países del Mercosur u obliga a las empresas de estos países a establecer acuerdos con firmas brasileñas que tengan productos ya registrados ante la autoridad sanitaria (De Queiroz, 1993).

En cuanto a la nueva legislación sobre patentes, en Argentina ella no tiene carácter retroactivo, es decir, no otorga derechos sobre productos que con anterioridad a 1995 (fecha de su entrada en vigencia) circulasen en el país y/o tuviesen patentes ya otorgadas en otros países. Por otra parte, las nuevas invenciones de productos farmacéuticos no serían patentables en la Argentina antes de transcurridos cinco años de publicada la ley (Challú y Lewis, 1996). A partir de entonces los titulares de las patentes tendrán durante 20 años el monopolio de los derechos de explotación, siempre que dicha explotación se haga efectiva, ya que cuando una patente no es utilizada las autoridades quedan facultadas para otorgar derechos de explotación a terceros. Se considera como "explotación" la adecuada distribución y comercialización del producto resultante de la patente en cuestión, lo que significa que dicha explotación se puede realizar tanto a través de la producción interna como de la importación. ${ }^{7}$

En Brasil, la ley de patentes tiene vigencia desde el momento de su puesta en vigor (mayo de 1996) y también plantea que la explotación debe hacerse efectiva, pero a diferencia de la ley argentina considera como "explotación" exclusivamente al abastecimiento interno por medio de la producción interna de los productos terminados. Esto significa que si una firma quiere tener el derecho a la explotación monopólica de su invención, se verá obligada a fabricar dicho producto en Brasil. Esta obligación de producción nacional ha generado ya un pedido de panel de los Estados Unidos, es decir, una solicitud para que se juzgue el caso en la Organización Mundial de Comercio (OMC). ${ }^{8}$

\footnotetext{
${ }^{7}$ Hacia principios de 2000, año en que debía entrar en vigencia en Argentina el pago de las patentes, se produjo en ese país un fuerte debate acerca de si el requisito de explotación de la patente debía implicar la producción interna del medicamento, como en Brasil.

${ }^{8}$ Pero Brasil, a su vez, acaba de denunciar que Estados Unidos establece una obligación similar para una serie de drogas y recientemente ganó una votación (por 53 a 1) en la Comisión de Derechos Humanos, condenando el uso excesivo de sus derechos de patentes por parte de ciertas empresas farmacéuticas. Por otro lado, ante la presión internacional un grupo de estas últimas ha abandonado recientemente un juicio contra Sudáfrica por el uso de drogas fundamentales para combatir la enfermedad causada por el VIH. Todo esto hace que la discusión sobre el derecho de patentes pueda asumir nuevas características en el futuro (Watal y Mathai, 1995).
} 
Estas diferencias entre Argentina y Brasil respecto a la ley de patentes pueden resultar decisivas en las estrategias de las firmas, incentivando su radicación en Brasil. En efecto, pueden permitir que el abastecimiento desde este país asegure al titular de una patente el monopolio en ambos países (dado que Argentina considera la importación como explotación), mientras que si se radica en Argentina puede encontrarse obligada a ceder una licencia para la fabricación en Brasil. Por otra parte, la diferencia entre las fechas de entrada en vigencia de ambas leyes (en lo referente a productos farmacéuticos) podría actuar en la misma dirección durante el período de transición en Argentina, para aquellos casos de radicaciones destinadas a explotar invenciones propias. En estos casos, la producción en Brasil al menos asegura el monopolio en dicho país, mientras que la fabricación en Argentina no lo hace en ninguno.

Otro aspecto que conviene destacar es que en ambos países ha habido intentos de promover las ventas a través de medicamentos genéricos, pero queda aún un largo camino por recorrer en este sentido. En Argentina se dispuso la confección de listados de mar- cas comerciales de medicamentos agrupadas según sus nombres genéricos, y la obligación de los médicos de prescribir utilizando dichos nombres. No obstante, esta última medida resultó difícil de aplicar y por un decreto posterior se dejó a criterio del médico la forma de prescripción. En Brasil se estableció en febrero de 1999 la exigencia de que todos los medicamentos comercializados a través de sus marcas muestren claramente en sus envases la denominación del fármaco o principio activo con que fue elaborado. ${ }^{9}$

Por otro lado, dentro del proceso de armonización de normativas que está teniendo lugar en el Mercosur se ha avanzado en aquellas vinculadas con la inspección de la calidad de los medicamentos, con las prácticas adecuadas para la fabricación de los mismos (GMP), con la inspección de plantas y la confección de guías a tal efecto, y con la capacitación de los inspectores. Estas armonizaciones permiten avanzar hacia el reconocimiento mutuo entre agencias gubernamentales de ambos países encargadas de autorizar las ventas de especialidades medicinales en sus respectivos mercados internos y han contribuido al intercambio y nivelación de conocimientos.

\section{III \\ Evolución reciente del sector farmacéutico en el Mercosur}

\section{Estructura y desempeño reciente}

El sector farmacéutico en los distintos países del Mercosur presenta algunos rasgos estructurales comunes.

En primer lugar, existe una clara división entre laboratorios trasnacionales y laboratorios nacionales. En Argentina y Uruguay, la facturación del sector se divide en partes más o menos iguales entre ambos tipos, mientras que en Brasil la relación es de $75 \%$ a $25 \%$ en favor de las trasnacionales. ${ }^{10}$ Las diferencias de propiedad se traducen en comportamientos micro-

\footnotetext{
${ }^{9}$ Con el propósito de avanzar en este proceso se le otorgó un plazo de 90 días a la Agencia de Vigilancia Sanitaria brasileña para establecer los criterios que determinen la equivalencia terapéutica de los medicamentos genéricos y las excepciones en su aplicación por decisión expresa del profesional prescriptor.

${ }^{10}$ En el caso uruguayo se incluyen en el conjunto de laboratorios nacionales aquellos de carácter binacional o multilatinos.
}

económicos diferentes: los laboratorios nacionales generalmente no hacen investigación científica y tecnológica, y sobreviven mediante la copia de medicamentos desarrollados en el exterior; los laboratorios trasnacionales, en cambio, aplican al mercado local las innovaciones realizadas en los centros mundiales de investigación. En ambos casos el origen de los principios activos (drogas) es fundamentalmente importado.

En segundo lugar, si bien el mercado farmacéutico en su conjunto se halla relativamente poco concentrado (las diez principales firmas no superan el $40 \%$ de las ventas totales en Argentina y Brasil), dentro de cada especialidad terapéutica el nivel de concentración es sensiblemente mayor. ${ }^{11}$ La promoción (en gran

\footnotetext{
${ }^{11}$ A modo de ejemplo para el caso brasileño, se puede apreciar que la participación del laboratorio líder en el mercado del genérico paracetamol llega al 91\%, en amoxicilina al 58\% y en dipirona al 93\%.
} 
CUADRO 1

Producción y consumo aparente de productos

farmacéuticos en Argentina, Brasil y Uruguay

(Millones de dólares corrientes)

\begin{tabular}{|c|c|c|c|c|c|c|c|c|c|}
\hline \multirow[b]{2}{*}{ Años } & \multicolumn{3}{|c|}{ Argentina } & \multicolumn{3}{|c|}{ Brasil } & \multicolumn{3}{|c|}{ Uruguay } \\
\hline & Producción & $\begin{array}{c}\text { Balance } \\
\text { comercial } \\
\text { (expo.-impo) }\end{array}$ & $\begin{array}{l}\text { Consumo } \\
\text { aparente }\end{array}$ & Producción & $\begin{array}{c}\text { Balance } \\
\text { comercial } \\
\text { (expo-impo) }\end{array}$ & $\begin{array}{l}\text { Consumo } \\
\text { aparente }\end{array}$ & Producción & $\begin{array}{c}\text { Balance } \\
\text { comercial } \\
\text { (expo-impo) }\end{array}$ & $\begin{array}{r}\text { Consumo } \\
\text { aparente }\end{array}$ \\
\hline 1988 & 1197 & -29 & 1226 & $\ldots$ & $\ldots$ & $\ldots$ & $\ldots$ & $\ldots$ & $\ldots$ \\
\hline 1989 & 927 & -24 & 951 & 7565 & -62 & 7606 & $\ldots$ & $\ldots$ & $\ldots$ \\
\hline 1990 & 1441 & -21 & 1462 & 10973 & -157 & 11276 & $\ldots$ & $\ldots$ & $\ldots$ \\
\hline 1991 & 2087 & -45 & 2132 & 8769 & -159 & 9090 & 146 & -14 & 160 \\
\hline 1992 & 2468 & -107 & 2575 & 9767 & -117 & 10009 & 147 & -26 & 173 \\
\hline 1993 & 3005 & -135 & 3140 & 12058 & -176 & 12476 & 185 & -41 & 236 \\
\hline 1994 & 3427 & -235 & 3662 & 14338 & -339 & 14993 & 183 & -50 & 233 \\
\hline 1995 & 3396 & -204 & 3600 & 17040 & -406 & 17964 & 193 & -57 & 250 \\
\hline 1996 & 3423 & -221 & 3644 & 17540 & -701 & 18575 & 201 & -75 & 276 \\
\hline 1997 & 3606 & -234 & 3840 & 19700 & -1170 & 20905 & $\ldots$ & $\ldots$ & $\ldots$ \\
\hline 1998 & $\ldots$ & $\ldots$ & $\ldots$ & 20313 & $\ldots$ & 21643 & $\ldots$ & $\ldots$ & $\ldots$ \\
\hline
\end{tabular}

Fuentes: Para Argentina: sobre ventas, la Cámara Argentina de Especialidades Medicinales (CAEME); sobre comercio exterior, el Instituto Nacional de Estadísticas y Censos (INDEC). Para Brasil: sobre producción y consumo aparente, la Fundación Comercio Exterior (FUNCEX); los datos de producción incluyen al sector de perfumería e higiene; sobre balance comercial: elaboración propia con datos de la Base INTAL. Para Uruguay: informe de Uruguay con datos del Instituto Nacional de Estadística (INE).

medida a través de los visitadores médicos) y la marca constituyen las principales formas de competencia, mientras que la competencia por precios sólo adquiere mayor importancia en las ventas directas a los sistemas públicos de salud (hospitales y otros).

En la estructura por tamaño de las firmas existen importantes diferencias entre los distintos países del Mercosur. En el caso brasileño, más del $50 \%$ de la facturación proviene de laboratorios grandes con más de 500 empleados. En el caso uruguayo, en cambio, la mayoría de las firmas son pequeñas y, hacia 1997 , sólo un laboratorio tenía más de 100 empleados.

La estructura de la demanda en los tres países estudiados también parece diferir significativamente. En Brasil, la demanda está constituida por casi mil empresas distribuidoras y 46 mil farmacias. Las empresas distribuidoras tienen actuación regionalizada y sólo en los últimos años han empezado a nacionalizar sus servicios. En cambio, en Argentina la colocación de medicamentos está concentrada en tres grandes distribuidoras y en ellas participan activamente los laboratorios más importantes. Dichas distribuidoras han avanzado hacia el segmento de las droguerías, incrementando el grado de concentración en la distribución.

La producción y el consumo aparente de productos farmacéuticos, medidos en dólares corrientes, muestran una tendencia de largo plazo al crecimiento, fundamentalmente en Argentina y Brasil, como puede verse en el cuadro 1. En 1966 la producción alcanzó en los tres países estudiados los 21 mil millones de dólares corrientes, de los cuales Brasil aportó un $82 \%$, Argentina un $17 \%$ y Uruguay el restante $1 \% .^{12}$

Ahora bien, los incrementos en los niveles de producción medidos en dólares corrientes reflejan no sólo cambios en la producción física sino también en el tipo de cambio real y en los niveles de precios de los productos farmacéuticos. Si se miden esos incrementos en precios constantes, el escenario cambia radicalmente: sólo la industria farmacéutica brasileña muestra crecimiento en el volumen físico de producción a lo largo de la década de 1990. Dicho crecimiento tiene lugar luego de la implementación del Plan Real y alcanza entre 1994 y 1998 al 23.6\% (igualmente muy lejos del $42 \%$ de crecimiento de la producción en dólares corrientes registrado en igual período). En el caso argentino, las ventas en unidades experimentaron una retracción del orden del 10\% entre 1991 y 1997, por lo que todo el incremento de producción medido en dólares corrientes se originó en el fuerte aumento de los precios medios de la industria (Ministerio de Economía de la Provincia de Buenos Aires, 1997).

\footnotetext{
12 Cabe señalar que las cifras de producción de Brasil incluyen al sector de perfumería e higiene, por lo que la participación de la industria farmacéutica brasileña en el MERCOSUR se halla sobrestimada.
} 
Este comportamiento indica que, a pesar del proceso de reducción arancelaria que tuvo lugar en los años noventa, la industria farmacéutica del Mercosur experimentó en dicha década una fuerte recuperación de sus precios relativos.

\section{Evolución del comercio de productos farma- céuticos en la década de 1990}

a) Exportaciones e importaciones globales

El comercio exterior de productos farmacéuticos del Mercosur exhibió un notable dinamismo en los años noventa, siendo mayor el crecimiento de las importaciones que el de las exportaciones (cuadros 2 y 3 ). ${ }^{13}$ Como resultado de esta dinámica el sector farmacéutico profundizó su déficit comercial, pasando de 204.3 millones de dólares en 1990 a 1022.1 millones en 1996 (cuadro 4).

Dentro de esta realidad global la participación que presentan los distintos países del Mercosur difiere de sus tamaños relativos tanto en lo referente a las exportaciones como a los valores brutos de producción, ya que Argentina y Uruguay tienen una participación relativamente elevada en las exportaciones totales, mientras que, en relación con su tamaño, la de Brasil es menor. En ese contexto, Argentina se perfila como el principal país exportador de la región (53\% de las exportaciones regionales en 1996) y Brasil como el principal mercado importador de ella (61\% de las importaciones del bloque para el mismo año).

En cuanto al grado de apertura comercial del sector, cabe señalar que la industria farmacéutica de los países del Mercosur es y sigue estando dirigida básicamente al mercado interno, como lo atestiguan los bajos coeficientes de exportación (cuadro 5). No obstante, en la década de 1990 las exportaciones tuvieron un comportamiento más dinámico que la producción, fundamentalmente en Argentina.

Los coeficientes de apertura a las importaciones son levemente mayores que los coeficientes de exportación. Aun así, y salvo en el caso uruguayo, la penetración importadora de productos farmacéuticos sigue siendo relativamente baja. Pero si se tomara en cuenta la provisión de materias primas (farmoquímica), estos coeficientes serían sustancialmente mayores.

En el patrón geográfico del comercio exterior de productos farmacéuticos del Mercosur existe una diferencia marcada entre las exportaciones y las importaciones. Las exportaciones dirigidas a la zona tienen una participación creciente en el total, ya que las correspondientes

CUADRO 2

Exportaciones de productos farmacéuticos del Mercosur

\begin{tabular}{|c|c|c|c|c|c|c|c|}
\hline & 1990 & 1991 & 1992 & 1993 & 1994 & 1995 & 1996 \\
\hline \multicolumn{8}{|c|}{ Miles de dólares } \\
\hline Argentina & 21569 & 44556 & 48258 & 73656 & 91357 & 131303 & 179058 \\
\hline Brasil & 43884 & 50652 & 66129 & 62332 & 83174 & 111164 & 131178 \\
\hline Uruguay & 6091 & 14142 & 14119 & 12116 & 13460 & 23636 & 21597 \\
\hline Paraguay & 156 & 452 & 1328 & 2004 & 3689 & 2987 & 4293 \\
\hline Mercosur & 71700 & 109802 & 129834 & 150108 & 191680 & 269090 & 336126 \\
\hline \multicolumn{8}{|c|}{ Porcentajes } \\
\hline Argentina & 30 & 41 & 37 & 49 & 48 & 49 & 53 \\
\hline Brasil & 61 & 46 & 51 & 42 & 43 & 41 & 39 \\
\hline Uruguay & 8.5 & 13 & 11 & 8 & 7 & 9 & 6 \\
\hline Paraguay & 0.5 & 0 & 1 & 1 & 2 & 1 & 1 \\
\hline Mercosur & 100 & 100 & 100 & 100 & 100 & 100 & 100 \\
\hline
\end{tabular}

Fuente: Elaboración propia sobre datos de la Base INTAL.

${ }^{13}$ Cabe señalar que los datos primarios surgen de las declaraciones de los distintos organismos oficiales de estadística de los países. Esto da lugar a problemas de confiabilidad y consistencia de las cifras de comercio exterior. Como ejemplo de ello se pueden citar los siguientes casos: las exportaciones fob de Argentina a Brasil del año 1989 (declaradas por Argentina) ascienden a 186 mil dólares, mientras que las importaciones cif de Brasil desde Argentina para el mismo año (declaradas por Brasil) alcanzan 1.8 millón de dólares; las exportaciones fob de Uruguay a Brasil en 1996 (declaradas por Uruguay) llegan a 10.1 millones de dólares, mientras que el valor cif declarado por Brasil asciende a 20 millones. En general, se aprecian fuertes variaciones entre los valores fob y cif declarados por los distintos países socios en el comercio intrazonal y estas diferencias no siempre se dan con el signo esperado. 
CUADRO 3

Importaciones de productos farmacéuticos del Mercosur

\begin{tabular}{|c|c|c|c|c|c|c|c|}
\hline & 1990 & 1991 & 1992 & 1993 & 1994 & 1995 & 1996 \\
\hline \multicolumn{8}{|c|}{ Miles de dólares } \\
\hline Argentina & 42322 & 89601 & 155955 & 207999 & 362362 & 335596 & 400896 \\
\hline Brasil & 200903 & 209423 & 183126 & 238692 & 422086 & 517319 & 832378 \\
\hline Uruguay & 21310 & 27397 & 36676 & 43460 & 54740 & 66659 & 79763 \\
\hline Paraguay & 11499 & 18962 & 23545 & 29038 & 50954 & 38913 & 45184 \\
\hline Mercosur & 276034 & 345383 & 399302 & 519189 & 854142 & 958487 & 1358221 \\
\hline \multicolumn{8}{|c|}{ Porcentajes } \\
\hline Argentina & 15 & 26 & 39 & 40 & 38 & 35 & 30 \\
\hline Brasil & 73 & 61 & 46 & 46 & 49 & 54 & 61 \\
\hline Uruguay & 8 & 8 & 9 & 8 & 6 & 7 & 6 \\
\hline Paraguay & 4 & 5 & 6 & 6 & 6 & 4 & 3 \\
\hline Mercosur & 100 & 100 & 100 & 100 & 100 & 100 & 100 \\
\hline
\end{tabular}

Fuente: Elaboración propia sobre datos de la Base INTAL.

CUADRO 4

Balance comercial de productos farmacéuticos del Mercosur (Miles de dólares)

\begin{tabular}{lrrrrrrrr}
\hline & 1990 & 1991 & 1992 & 1993 & 1994 & 1995 \\
\hline Argentina & -20753 & -45045 & -107697 & -134343 & -235005 & -204293 \\
Brasil & -157019 & -158771 & -116997 & -176360 & -338912 & -406155 & -221838 \\
Uruguay & -15219 & -13255 & -22557 & -31344 & -41280 & -43023 & -58166 \\
Paraguay & -11343 & -18510 & -22217 & -27034 & -47265 & -35926 & -40891 \\
Mercosur & -204334 & -235581 & -269468 & -369081 & -662462 & -689397 & -1022095 \\
\hline
\end{tabular}

Fuente: Elaboración propia sobre datos de la Base INTAL.

CUADRO 5 Coeficientes de exportación
(Porcentajes)

\begin{tabular}{lccc}
\hline & Brasil & Argentina & Uruguay \\
\hline 1991 & 1 & 2 & 10 \\
1992 & 1 & 2 & 10 \\
1993 & 1 & 2 & 7 \\
1994 & 1 & 3 & 7 \\
1995 & 1 & 4 & 12 \\
1996 & 1 & 5 & 11 \\
1997 & 3 & 7 & $\ldots$ \\
\hline
\end{tabular}

Fuente: Informes nacionales de Argentina, Brasil y Uruguay para los datos sobre producción. Los datos sobre comercio son de elaboración propia con información de la Base INTAL, salvo para Brasil en 1997, que se obtuvo del correspondiente informe nacional.

al resto del mundo crecen a una tasa sensiblemente menor y se concentran en los países en desarrollo, especialmente los de América Latina (cuadro 6).

Las importaciones, en cambio, muestran (cuadro 7) que, aunque la participación de la zona ha crecido en los últimos años, en 1996 la de Brasil y Argentina apenas llegó al 10 ó $12 \%$ del total. Esto quiere decir que el grueso de las importaciones sigue proviniendo de los países desarrollados (Estados Unidos y Europa).

b) Indice de ventajas comparativas reveladas

En el decenio de 1990 los países del Mercosur se vieron afectados, en mayor o menor medida, por marcadas fluctuaciones macroeconómicas que tuvieron un fuerte impacto tanto sobre el resultado de su balance comercial global como sobre los correspondientes a sus distintos sectores productivos. Es por ello que aquí emplearemos el indicador de ventaja comparativa revelada $^{14}$ (también llamado índice de contribución al

\footnotetext{
${ }^{14}$ El índice de ventajas comparativas reveladas se construye restando al balance comercial sectorial un valor teórico que representa la parte que le corresponde al sector del balance comercial general. Está expresado en milésimos del comercio total y su valor puede oscilar entre -500 y +500 (en los casos de una especialización completa). La fórmula del indicador es: VCR $=((\mathrm{Xi}-\mathrm{Mi})-(\mathrm{Xi}+\mathrm{Mi}) /$ $(\mathrm{X}+\mathrm{M}) *(\mathrm{X}-\mathrm{M})) * 1000 /(\mathrm{X}+\mathrm{M})$.
} 
CUADRO 6

Destino de las exportaciones de Argentina, Brasil y Uruguay

(Porcentajes)

\begin{tabular}{llccccccc}
\hline & Destino/años & 1990 & 1991 & 1992 & 1993 & 1994 & 1995 & 1996 \\
\hline Argentina & Mercosur & 44 & 41 & 48 & 49 & 56 & 53 & 61 \\
& Resto del mundo & 56 & 59 & 52 & 51 & 44 & 47 & 39 \\
\multirow{3}{*}{ Brasil } & Mercosur & 8 & 14 & 20 & 22 & 27 & 37 & 41 \\
& Resto del mundo & 92 & 86 & 80 & 78 & 73 & 63 & 59 \\
\multirow{3}{*}{ Uruguay } & Mercosur & 28 & 70 & 55 & 58 & 56 & 70 & 58 \\
& Resto del mundo & 72 & 30 & 45 & 42 & 44 & 30 & 42 \\
\hline
\end{tabular}

Fuente: Elaboración propia sobre datos de la Base INTAL.

CUADRO 7

Origen de las importaciones de Argentina, Brasil y Uruguay

(Porcentajes)

\begin{tabular}{llrrrrrrr}
\hline & Destino/años & 1990 & 1991 & 1992 & 1993 & 1994 & 1995 & 1996 \\
\hline \multirow{2}{*}{ Argentina } & Mercosur & 1 & 4 & 6 & 6 & 5 & 12 & 12 \\
& Resto Mundo & 99 & 96 & 94 & 94 & 95 & 88 & 88 \\
\multirow{3}{*}{ Brasil } & Mercosur & 1 & 5 & 6 & 5 & 6 & 8 & 10 \\
& Resto Mundo & 99 & 95 & 94 & 95 & 94 & 92 & 90 \\
\multirow{2}{*}{ Uruguay } & Mercosur & 28 & 40 & 36 & 38 & 42 & 41 & 42 \\
& Resto Mundo & 72 & 60 & 64 & 62 & 58 & 59 & 58 \\
\hline
\end{tabular}

Fuente: Elaboración propia sobre datos de la Base INTAL.

CUADRO 8

Indice de ventajas comparativas reveladas en el sector farmacéutico del Mercosur (Milésimos del comercio total)

\begin{tabular}{lllllll}
\hline & 1990 & 1991 & 1992 & 1993 & 1994 & 1995 \\
\hline Argentina & -3.22 & -3.44 & -3.20 & -3.36 & -4.56 & -5.23 \\
Brasil & -3.99 & -4.01 & -3.28 & -3.46 & -4.93 & -4.68 \\
Uruguay & -5.71 & -4.35 & -5.35 & -5.47 & -6.88 & -7.72 \\
Paraguay & -4.68 & -6.62 & -7.65 & -7.47 & -7.91 & -3.60 \\
Mercosur & -3.99 & -4.05 & -3.83 & -4.03 & -5.36 & -4.45 \\
\hline
\end{tabular}

Fuente: Elaboración propia sobre datos de la Base INTAL.

saldo) como forma de separar dentro de los saldos sectoriales los efectos de los cambios en los balances comerciales globales.

El cuadro 8 indica que el Mercosur presenta, en el sector farmacéutico, una desventaja comparativa revelada con el resto del mundo cuyo nivel se profundizó a lo largo de los años noventa. Esto significa que el déficit comercial normalizado del sector (descontado el efecto de los déficit o superávit globales del comercio) se ha incrementado con relación al volumen total de comercio de la región. De ello puede deducirse que en el patrón de comercio han ocurrido cambios estructurales que no dependen exclusivamente de la modificación en las coyunturas macroeconómicas que tuvo lugar en la década de 1990.

Ahora bien, este comportamiento agregado del sector farmacéutico se corresponde en forma más o menos similar con los casos individuales de los países del Mercosur. En todos ellos las exportaciones e importaciones se expanden a tasas altas y el déficit comercial 
sectorial y la desventaja comparativa revelada tienden a profundizarse.

\section{Comercio intrazonal en el Mercosur}

Fruto del proceso de integración regional, el comercio intrazonal ha tenido un dinamismo aun mayor que el extrazonal. En efecto, durante el período considerado, las exportaciones intrazonales se han incrementado en un $1110 \%$, de 14.6 miles de dólares en 1990 a 176.7 miles de dólares en 1996 (cuadros 9 y 10).

En las exportaciones intrazonales se puede apreciar que el liderazgo exportador de Argentina es aun más pronunciado que en las exportaciones a todo el mundo (lo que evidencia una mayor dependencia de Argentina del mercado zonal). Asimismo, por el lado de las importaciones puede apreciarse un peso relativamente mucho mayor de Uruguay y Paraguay (es decir, ambos se abastecen en una proporción relativamente mayor en los países del Mercosur).

Este comportamiento de los flujos comerciales determina un patrón de comercio intrazonal donde Argentina aparece crecientemente como el único país superavitario, mientras que Uruguay, Paraguay y, a partir de 1994, Brasil tienen un balance deficitario (cuadro 11).

El mayor dinamismo de las exportaciones intrazonales respecto de las dirigidas al resto del mundo tra-

CUADRO 9

Exportaciones intrazonales de productos farmacéuticos en el Mercosur

\begin{tabular}{|c|c|c|c|c|c|c|c|}
\hline & 1990 & 1991 & 1992 & 1993 & 1994 & 1995 & 1996 \\
\hline \multicolumn{8}{|c|}{ Miles de dólares } \\
\hline Argentina & 9553 & 18138 & 23025 & 36170 & 50836 & 69056 & 108584 \\
\hline Brasil & 3385 & 7038 & 13343 & 13390 & 22197 & 41280 & 53735 \\
\hline Uruguay & 1692 & 9927 & 7814 & 7040 & 7502 & 16642 & 12614 \\
\hline Paraguay & 16 & 281 & 684 & 1187 & 2515 & 828 & 1772 \\
\hline Mercosur & 14646 & 35384 & 44866 & 57787 & 83050 & 127806 & 176705 \\
\hline \multicolumn{8}{|c|}{ Porcentajes } \\
\hline Argentina & 65 & 51 & 51 & 63 & 61 & 54 & 61 \\
\hline Brasil & 23 & 20 & 30 & 23 & 27 & 32 & 30 \\
\hline Uruguay & 12 & 28 & 17 & 12 & 9 & 13 & 7 \\
\hline Paraguay & 0 & 1 & 2 & 2 & 3 & 1 & 1 \\
\hline Mercosur & 100 & 100 & 100 & 100 & 100 & 100 & 100 \\
\hline
\end{tabular}

Fuente: Elaboración propia sobre datos de la Base INTAL.

CUADRO 10

Importaciones intrazonales de productos farmacéuticos en el Mercosur

\begin{tabular}{|c|c|c|c|c|c|c|c|}
\hline & 1990 & 1991 & 1992 & 1993 & 1994 & 1995 & 1996 \\
\hline \multicolumn{8}{|c|}{ Miles de dólares } \\
\hline Argentina & 626 & 3315 & 9809 & 12484 & 17426 & 39648 & 48915 \\
\hline Brasil & 2310 & 9582 & 10244 & 12313 & 24227 & 42765 & 79435 \\
\hline Uruguay & 6056 & 10882 & 13207 & 16586 & 22793 & 27176 & 33659 \\
\hline Paraguay & 6460 & 10099 & 12370 & 16870 & 19434 & 22554 & 29169 \\
\hline Mercosur & 15452 & 33878 & 45630 & 58253 & 83880 & 132143 & 191178 \\
\hline \multicolumn{8}{|c|}{ Porcentajes } \\
\hline Argentina & 4 & 10 & 21 & 21 & 21 & 30 & 26 \\
\hline Brasil & 15 & 28 & 22 & 21 & 29 & 32 & 42 \\
\hline Uruguay & 39 & 32 & 29 & 28 & 27 & 21 & 18 \\
\hline Paraguay & 42 & 30 & 27 & 29 & 23 & 17 & 15 \\
\hline Mercosur & 100 & 100 & 100 & 100 & 100 & 100 & 100 \\
\hline
\end{tabular}

Fuente: Elaboración propia sobre datos de la Base INTAL. 
CUADRO 11

Balance del comercio intrazonal de productos farmacéuticos en el Mercosur (Miles de dólares)

\begin{tabular}{lrrrrrrrr}
\hline & 1990 & 1991 & 1992 & 1993 & 1994 & 1995 \\
\hline Argentina & 8927 & 14823 & 13216 & 23686 & 33410 & 29408 \\
Brasil & 1075 & -2544 & 3099 & 1077 & -2030 & -1485 & -25700 \\
Uruguay & -4364 & 955 & -5393 & -9546 & -15291 & -10534 & -21045 \\
Paraguay & -6444 & -9818 & -11686 & -15683 & -16919 & -21726 \\
\hline
\end{tabular}

Fuente: Elaboración propia con datos de la Base INTAL.

CUADRO 12

Indice de orientación zonal en el sector farmacéutico

\begin{tabular}{|c|c|c|c|c|c|c|c|}
\hline & 1990 & 1991 & 1992 & 1993 & 1994 & 1995 & 1996 \\
\hline Argentina & 4.56 & 3.47 & 3.88 & 2.47 & 2.88 & 2.33 & 3.09 \\
\hline Brasil & 1.90 & 2.05 & 1.96 & 1.78 & 2.31 & 3.87 & 3.84 \\
\hline Uruguay & 0.72 & 4.29 & 2.49 & 2.00 & 1.43 & 2.68 & 1.52 \\
\hline Paraguay & 0.17 & 3.03 & 1.77 & 2.21 & 3.00 & 0.29 & 0.41 \\
\hline
\end{tabular}

Fuente: Elaboración propia sobre datos de la Base INTAL.

duce una tendencia general del comercio de los países socios del Mercosur. El índice de orientación zonal ${ }^{15}$ (cuadro 12) nos señala que, en los casos de Argentina y Brasil, las exportaciones farmacéuticas muestran una orientación zonal sustancialmente mayor que la unidad. Es decir, las exportaciones farmacéuticas de Argenti- na ${ }^{16} \mathrm{y}$ de Brasil dependen de los mercados zonales relativamente más que las exportaciones totales de cada uno de dichos países. Sin embargo, en Brasil esa orientación zonal muestra una tendencia creciente a lo largo de la década, mientras que en Argentina la tendencia es decreciente.

\section{IV}

\section{Efectos estáticos y dinámicos de la apertura comercial, la integración y los cambios regulatorios sobre el sector farmacéutico}

La industria farmacéutica, tanto a nivel internacional como en el zonal, está sufriendo un proceso de profundas transformaciones. Las empresas del sector han debido adaptarse a nuevas formas de llevar adelante sus procesos de investigación científica y tecnológica, nue-

\footnotetext{
15 El índice de orientación zonal compara la importancia relativa que tiene el mercado regional para las exportaciones sectoriales con su importancia para las exportaciones totales del país. El indicador oscila entre cero e infinito. El valor unitario sugiere la ausencia de orientación regional. La fórmula correspondiente es: IORi $=\mathrm{Xij} / \mathrm{Xi} / \mathrm{Xj} / \mathrm{X}$.
}

vos regímenes regulatorios (basados fundamentalmente en la liberación comercial y la eliminación del control de precios), nuevas señales de mercado y nuevos

\footnotetext{
${ }^{16}$ La existencia de una orientación zonal positiva en el caso argentino se debe al hecho de que gran parte de las exportaciones primarias de este país se dirigen al resto del mundo. Si en vez de tomar como referencia las exportaciones totales se utilizaran las exportaciones de manufacturas de origen industrial, el resultado sería inverso. Es decir, las exportaciones farmacéuticas dependen más del mercado regional que las exportaciones primarias, pero menos que el promedio de las manufacturas de origen industrial.
} 
patrones de relación con los entes públicos y privados. Es decir, la profundización del proceso de integración regional a través del Mercosur ha coincidido con otros cambios estructurales que han tenido lugar en la región. Esto nos plantea la dificultad metodológica de identificar cuáles aspectos del proceso de transformación sectorial comentados anteriormente pueden ser atribuidos a los efectos de la integración regional y cuáles obedecen al impacto de los demás fenómenos comentados. En las subsecciones siguientes intentaremos responder a este interrogante en lo referente a los efectos de carácter tanto estático como dinámico.

\section{Análisis de los efectos estáticos}

Los análisis de tipo estático de los procesos de integración y apertura generalmente apuntan a dilucidar en qué medida dichos procesos reducen las distorsiones estáticas inducidas por la protección comercial. Estos análisis tienden a centrarse en el comportamiento de las importaciones y en la forma en que ellas desplazan la producción nacional ineficiente, pero también involucran la evaluación de la tendencia de los precios internos y su efecto en el bienestar del consumidor.

Desde dicha perspectiva sobre el comportamiento del comercio, es indudable que tanto los procesos de apertura comercial como los de integración regional han generado beneficios estáticos en la zona. Las importaciones totales de los tres países considerados han aumentado, proporcionalmente, mucho más que sus respectivos niveles de consumo aparente. Esto incrementó marcadamente el desequilibrio sectorial de la zona con el resto del mundo y elevó los coeficientes de importación (si bien éstos siguen siendo aún relativamente bajos). Se produjo, en consecuencia, una caída gradual del índice de ventajas comparativas reveladas de la zona frente al resto del mundo, que se corresponde en forma más o menos similar para los cuatro países de la región.

Por otro lado, el proceso de integración hizo posible un notable crecimiento del comercio intrazonal. Tanto las importaciones como las exportaciones superan en más de 10 veces los niveles de fines de los años ochenta. El hecho de que las importaciones extrazonales también hayan aumentado y que los niveles de producción muestren crecimientos mucho menores nos permite suponer la existencia de un importante proceso de creación de comercio en la zona.

El patrón de comercio de la zona nos muestra hoy que el grueso de sus importaciones sigue proviniendo de los países desarrollados, mientras que el aumento que tuvo lugar en las exportaciones al Mercosur convierte a éste en el principal destinatario de ellas.

El incremento de las exportaciones zonales es protagonizado por Argentina, que consolida su liderazgo exportador en el Mercosur y que se mantiene como único país con balance sectorial positivo. Pero a pesar del fuerte avance que muestra Brasil como mercado para las exportaciones argentinas, este hecho beneficia fundamentalmente a las empresas trasnacionales del sector, ya que las de capital nacional se orientan más hacia los mercados de Uruguay y Paraguay. ${ }^{17}$ Esto es atribuido por las empresas argentinas a las asimetrías de regulación existentes entre Argentina y Brasil y al hecho de que para exportar a Brasil se requiere realizar una gran inversión destinada a imponer comercialmente las marcas propias.

En Brasil las empresas trasnacionales también tienen un papel protagónico en las exportaciones. Allí, de las 10 principales empresas exportadoras al Mercosur (que representan alrededor del $70 \%$ de las exportaciones) ocho son trasnacionales y dos son establecimientos "no clasificados", mientras que los principales laboratorios nacionales no están entre los mayores exportadores a la zona. Las empresas trasnacionales figuran también entre las principales empresas importadoras de productos del Mercosur. Un conjunto de empresas trasnacionales actúan a la vez como importadoras y exportadoras de productos farmacéuticos, reflejando una estrategia de especialización intraindustrial.

En la composición del comercio intrazonal parece delinearse un patrón de especialización caracterizado por exportaciones de medicamentos a granel e importaciones de medicamentos al por menor por parte de Uruguay y una especialización intraindustrial entre Argentina y Brasil en medicamentos al por menor (con una participación importante de antibióticos, hormonas y enzimas, antiinflamatorios y otros).

En conclusión, a partir del incremento de las preferencias arancelarias en el Mercosur se ha desarrollado claramente un mayor dinamismo de las exportaciones intrazonales que de las dirigidas al resto del mundo, dinamismo que es claramente liderado por las empresas trasnacionales. Pero esto va acompañado por una tendencia ascendente de las importaciones desde el resto del mundo, lo que no nos permite inferir la existencia de desvío de comercio y la que, además,

\footnotetext{
17 También hacia otros países latinoamericanos, especialmente en aquellos en que los laboratorios nacionales poseen plantas propias.
} 
produce una leve caída en el índice de comercio intraindustrial y una tendencia errática en el índice de orientación regional, a lo largo de la década.

Las empresas nacionales de todos los países considerados plantean la existencia de asimetrías regulatorias que dificultan el aprovechamiento de las ventajas comparativas existentes o plantean riesgos de excesiva penetración importadora. Las empresas argentinas señalan que las restricciones de acceso al registro de medicamentos en Brasil hacen que sólo las trasnacionales puedan beneficiarse con la expansión de las exportaciones dirigidas a ese país. Es que las firmas locales deben pasar por un fuerte control de calidad en plantas y productos, hecho que limita el ingreso de sus bienes al país vecino. Las empresas brasileñas, en cambio, alegan que las reglas de control sanitario son más blandas en Argentina, ya que las exigencias del licenciamiento y control de calidad de los medicamentos son menores, y que esto afecta la competitividad del producto brasileño. Otro efecto estático de la apertura comercial en Argentina y Brasil ha sido el fuerte aumento de las importaciones de productos farmoquímicos, lo que ha contribuido a reducir considerablemente los costos de producción de la industria farmacéutica.

Resulta interesante destacar que los aparentes efectos estáticos positivos que surgen del comportamiento de los flujos comerciales plantean una fuerte contradicción con el comportamiento de los precios internos. En efecto, los precios internos de los medicamentos de los tres países considerados se fueron elevando a lo largo de todo el decenio de 1990.

En el caso argentino, en particular, las ventas en unidades permanecieron estancadas ( $\mathrm{y}$ hasta cayeron levemente según los años de comparación), mientras que los precios medios en dólares exhibieron un incremento constante que alcanzó el 100\% entre 1991 y 1997 y un $240 \%$ si se toma como punto de comparación el año 1988 (datos del CAEME). Esto revela que los consumidores se han visto muy perjudicados por la dinámica que han generado las distintas transformaciones ocurridas en el sector. La tendencia indicada al incremento de los precios se retroalimenta negativamente con características específicas del mercado de la salud, como la diferenciación entre quién decide el gasto - el médico- y quién debe afrontarlo, o la existencia de seguros de salud más o menos generalizados. Tales características tienden a provocar una utilización desmedida de medicamentos y falta de capacidad de negociar los precios en quienes financian los gastos (fundamentalmente los consumidores).

\section{Efectos dinámicos del proceso de integración}

Como se vio en la sección III, el sector farmacéutico de la zona está atravesando un profundo proceso de transformación. Esto está originando fuertes cambios en las formas de desarrollar, fabricar y comercializar medicamentos, a partir de modificaciones en las conductas y estrategias de las empresas e instituciones del sector. Como veremos a continuación, la estrategia varía considerablemente según se trate de empresas de capital nacional o de subsidiarias de empresas extranjeras.

¿En qué medida ha influido el proceso de integración de la zona en esta transformación? Tanto en el caso de Argentina como en el de Brasil, la importancia asignada por las empresas a la integración como detonante de las transformaciones ocurridas ha sido menor que la asignada al cambio en las regulaciones (precios máximos, apertura, ley de patentes) y a la estabilización macroeconómica. A continuación se describen algunas de las áreas donde cabría haber esperado efectos dinámicos positivos del proceso de integración.

a) Presión competitiva y reconversión sectorial Como ya fue señalado, ni el proceso de integración ni el de apertura han generado, hasta el momento, una mayor presión competitiva sobre los precios internos de los países del Mercosur. Esto puede ser atribuido a la estructura de mercado del sector en donde las especialidades medicinales presentan, en general, mercados cautivos por la marca — resultantes de fuertes campañas de promoción- en los cuales se está produciendo en algunos casos una fuerte concentración en lo que toca a la distribución. Por otro lado, la importación de medicamentos está controlada en muchos casos por los mismos laboratorios productores, por lo que su capacidad de disciplinar los precios se ve fuertemente limitada.

Pero si bien no se ha dado en la zona una presión competitiva por el lado de los precios internos, puede observarse claramente un nuevo escenario de competencia determinado por las importantes modificaciones efectuadas en las regulaciones, particularmente en lo que concierne a la ley de patentes. La profundidad de los cambios que plantea este nuevo escenario es tal que puede llegar a cuestionar la viabilidad de los laboratorios nacionales, particularmente de los más pequeños.

La entrada en vigor de las regulaciones sobre patentes reduce el espacio productivo de las empresas nacionales, pues limita sus posibilidades de participar 
en la fabricación de los nuevos productos que se van lanzando al mercado y que suelen ser los más rentables.

Frente a esta realidad las opciones que se abren a las empresas nacionales son las de establecer acuerdos con las firmas trasnacionales, participar crecientemente en el mercado de productos genéricos o vender sus plantas.

La estrategia de las empresas de mayor tamaño parece orientarse cada vez más a concretar nuevas formas de alianza estratégica con las firmas trasnacionales. Esto les permitiría tener acceso temprano a sus licencias mediante acuerdos de comercialización conjunta, o actuar como agentes de distribución local de moléculas y principios activos desarrollados por aquéllas en el exterior.

Con miras a mejorar su capacidad de negociación con las empresas extranjeras, una de las estrategias que está siendo crecientemente adoptada por los laboratorios nacionales de los distintos países es el refuerzo en sus estructuras de comercialización y la consolidación del prestigio de sus marcas y de la confianza en ellas.

La participación en el mercado de productos genéricos no es una solución obvia para las empresas en Argentina. Cuando se intentó imponer allí una legislación que promovía el uso de estos productos, ella fue fuertemente cuestionada por distintos sectores de la industria. Puesto que los productos genéricos requieren una industria moderna y alta escala de producción como forma de abaratar los costos, su desarrollo ha sido mayor en Brasil, por el gran tamaño de su mercado.

Esta realidad sugiere que la tendencia al aumento de la participación de las empresas extranjeras en los mercados de Brasil y Argentina se reforzará en el futuro. El proceso de integración ha reforzado el interés de las empresas trasnacionales por reposicionarse en esos mercados. El Mercosur les ofrece mayores posibilidades de expandir el comercio intrafirma debido a la liberalización arancelaria regional, a la cercanía geográfica y a la perspectiva de que en el futuro se alcance un alto nivel de armonización regulatoria entre los países miembros. La estrategia más común que están adoptando esas empresas en Argentina, y especialmente en Brasil, es la de revitalizar sus plantas fabriles, adquirir plantas existentes y establecer nuevas redes de distribución y comercialización.

En Uruguay, sin embargo, se está produciendo la situación opuesta. En efecto, las empresas trasnacionales en ese país están tendiendo a abandonar la producción local para vender exclusivamente medicamentos importados, particularmente desde Argentina. Algunas de las plantas que estas empresas han cerrado fueron o están siendo compradas por plantas nacionales o por empresas "multilatinas", es decir, por aquellas que tienen presencia en este país y en algún otro del Mercosur (Laens y Terra, 1998).

El reposicionamiento de las empresas trasnacionales en Argentina y Brasil se está realizando por diferentes caminos: mediante la adquisición de empresas que eran de capital nacional (Bristol Myers compró Argentia en Argentina) o la fusión con ellas (Hanssen y Cilag Farma en Argentina; Merck Sharp y Dohme y Ache, en Brasil ). Por otro lado, firmas que habían perdido dinamismo en el transcurso de los años ochenta recuperaron posiciones, reacondicionando y mejorando las plantas fabriles existentes (Rhodia Farma en Brasil). Un último grupo incorporó laboratorios nuevos o inició planes de expansión, construyendo plantas farmacéuticas (Bayer en Argentina, Glaxo Wellcome y Pfizer en Brasil). Esta reestructuración ha incluido el traslado de fábricas de un país a otro, como el cierre de plantas en Uruguay y también en Argentina (Alcon) para su relocalización en Brasil.

En consecuencia, con la salvedad de Uruguay, en los últimos años se ha estado modificando en la zona la participación de mercado según el origen del capital, en favor de los laboratorios trasnacionales. Sin embargo, se observa en éstos una escasa o nula intención de expandir la capacidad de producción de principios activos o materias primas farmacéuticos. Es más, a partir del proceso de apertura comercial, varias empresas trasnacionales abandonaron la producción de principios activos y optaron por una política de importación negociada intrafirma. Por su parte, las firmas nacionales también han ido sustituyendo la producción local de principios activos por su importación. ${ }^{18}$ Este proceso, que ha permitido a los laboratorios obtener un abaratamiento en el precio de las materias primas, ha agudizado fuertemente el déficit comercial de la industria farmoquímica de la zona.

\section{b) Especialización productiva y reducción de la gama de productos}

El proceso de integración del Mercosur está acentuando una tendencia originada en las estrategias de globalización de las empresas trasnacionales: les está posibilitando utilizar las ventajas desarrolladas por las

\footnotetext{
18 Este no es el caso de Uruguay, país que no ha desarrollado la producción de principios activos, por lo que las firmas farmacéuticas de ese país, que no visualizan a la zona como fuente de aprovisionamiento de materias primas, reclaman una reducción en el arancel externo común de los mismos.
} 
naciones para localizar entre ellas las diferentes líneas terapéuticas. Esto les permite reducir la gama de productos y aumentar la especialización productiva de sus distintas filiales, favoreciendo así un fuerte crecimiento de las relaciones comerciales intrafirma. Este proceso está llevando, por ejemplo, a que el laboratorio Glaxo especialice sus plantas argentinas en antibióticos y las de Brasil en tabletas y cremas, y a que Roche fabrique vitaminas en sus plantas argentinas e importe los productos inyectables desde Brasil.

En el caso de las firmas nacionales, los laboratorios argentinos encuestados están recurriendo a dos mecanismos para reducir su gama de productos. Por un lado están reduciendo la cantidad de productos que lanzan al mercado, lo que les permite concentrar los esfuerzos de investigación y comercialización. Por otro lado, varias empresas han establecido acuerdos de abastecimiento con otras firmas nacionales, para proveerse recíprocamente de distintos tipos de productos que luego venden con marcas propias, impulsadas por la necesidad de elevar la competitividad (vía economías de escala) y también por razones sanitarias (un laboratorio no puede producir distintas líneas de medicamentos).

Los laboratorios nacionales de Uruguay también están reduciendo su gama de productos, abandonando la producción de algunas líneas que han comenzado a importar desde Argentina y especializándose en otras para aprovechar economías de escala y marcas comunes en uno y otro país. En el caso de Brasil, son los laboratorios trasnacionales los que se pueden beneficiar al relocalizarse su producción entre los países del Mercosur.

\section{c) Estrategias exportadoras y alianzas empresaria- les regionales \\ Como ya se ha señalado, el Mercosur ha favore-} cido las relaciones comerciales entre las empresas trasnacionales de la zona, promoviendo la expansión del comercio intrafirma. Sólo en pocos casos se ha dado un aumento del comercio entre Argentina y Brasil a partir de exportaciones de empresas nacionales. Las empresas argentinas señalan que lograrlo se hace muy difícil por las restricciones a la certificación de productos en el registro brasileño y por la necesidad de hacer fuertes inversiones en comercialización. En este sentido, el impacto de la integración zonal en un desarrollo estratégico destinado a la exportación se ha hecho sentir fundamentalmente, hasta ahora, en las exportaciones hacia los mercados más pequeños de la zona. Esto se observa particularmente en aquellas empresas nacionales que tienen plantas en Argentina y Brasil, como Elea, Bagó y Raffo. Algunas de las empresas nacionales entrevistadas han señalado que tales exportaciones al Mercosur les han permitido desarrollar un proceso de aprendizaje de la actividad de exportación.

Según las empresas trasnacionales de Brasil que fueron entrevistadas, el Mercosur no ha alterado significativamente su actuación en los mercados de la zona porque su estrategia de negocios se basa, en mucho mayor medida, en las relaciones con la casa matriz. En cambio, las empresas brasileñas nacionales muestran una estrategia defensiva frente al Mercosur, que si bien atribuyen a un mercado con poco potencial de crecimiento, puede estar vinculada con su menor competitividad frente a las plantas argentinas.

Las restricciones señaladas por las empresas nacionales de los países del Mercosur hacen que para su expansión comercial en la zona necesiten un socio local en algún otro país dentro de ella. Hasta el momento prácticamente no se han establecido acuerdos comerciales o productivos entre empresas nacionales de Argentina y Brasil. Desde la perspectiva de las empresas argentinas, las posibilidades de asociación se encuentran limitadas por la relativa debilidad de las empresas de Brasil, mientras que estas últimas plantean una posición defensiva que, como ya fue señalado, atribuyen a asimetrías regulatorias que no las favorecen.

Sin embargo, en ambos países se han producido acuerdos comerciales liderados por empresas que han realizado avances tecnológicos propios. ${ }^{19}$

d) Desarrollo de capacidad tecnológica, captación de ciertos nichos de mercado y refuerzo de las estructuras de comercialización

Las empresas trasnacionales concentran la investigación básica fuera de la región. Mientras que la innovación en estas empresas está presente como estrategia mundial, parece orientarse más a nivel nacional hacia cambios organizacionales, especialmente referidos a la gestión empresarial y a la comercialización.

\footnotetext{
19 En Brasil la empresa Macrobiológica (que avanzó en la producción de principios activos) suscribió un acuerdo comercial con una empresa argentina para venderle materias primas. En Argentina, la empresa Bio Sidus (con avances propios en el campo de la biotecnología) ha concretado también un acuerdo comercial con una empresa brasileña y se ha convertido en el quinto exportador de productos farmacéuticos a Brasil, en abierta competencia con empresas trasnacionales, lo que le ha permitido aumentar su participación de mercado tanto en Brasil como en América Latina.
} 
Algunos laboratorios nacionales argentinos están realizando tareas de investigación aplicada y para ello han establecido acuerdos de colaboración con universidades y centros de investigación, con el incentivo de los créditos que otorga el Fondo Tecnológico Argentino. En unos pocos casos esos acuerdos han permitido, como ya fue señalado, realizar avances tecnológicos propios, particularmente en productos biotecnológicos. Sin embargo, los niveles de inversión en investigación científica y tecnológica son bajos y no se puede avanzar más allá de una determinada fase debido al alto costo que implica obtener registros en los países del norte.

Otras empresas nacionales argentinas están llevando adelante procesos de diferenciación de productos, en lo que se refiere tanto a las características del medicamento como al envase. En otros casos han tendido a insertarse en la producción destinada a ciertos nichos de mercado, como por ejemplo el de los productos fitoterápicos que no son patentables. Pero lo que puede desprenderse de las entrevistas realizadas es que, dado su menor nivel de madurez científico-tecnológica, las empresas nacionales, salvo excepciones, han optado por abandonar esfuerzos internos previos de investigación científica y tecnológica.

Las estrategias de las empresas nacionales argentinas y brasileñas aparecen muy condicionadas por la apertura económica y por la ley de patentes, la que establece limitaciones inmediatas (para las empresas brasileñas) o en un futuro cercano (para las argentinas) vinculadas al lanzamiento de nuevos productos. Esta realidad augura un futuro retroceso de su participación en los respectivos mercados nacionales.

\section{e) Algunas asignaturas pendientes del proceso de integración}

Lo que puede desprenderse de esta investigación es que la integración del Mercosur en el sector de productos farmacéuticos es aún un proceso muy incipiente, es decir, que la liberalización del comercio entre los países de la zona está lejos de ser una condición suficiente para constituir un mercado integrado. Entre las tareas pendientes para lograr tal objetivo y para potenciar el desarrollo de ventajas comparativas dinámicas frente a terceros mercados señalaremos las siguientes:

i) La eliminación de asimetrías de naturaleza regulatoria. Esto abarca un amplio espectro de políti- cas y acciones de los órganos nacionales de salud pública como la armonización de las reglas de licenciamiento de los productos y de certificación de productos bioequivalentes, las normas sanitarias requeridas para la importación de medicamentos, las regulaciones relativas a la comercialización de productos genéricos, etc. Incluye también armonizar políticas correspondientes a otros organismos públicos, como las vinculadas con la ley de patentes y con las políticas de competencia en el ámbito de la comercialización de productos.

ii) La promoción de acuerdos de cooperación tecnológica para impulsar el desarrollo de la región en este campo. En este sentido existe una antecedente dado por el Acuerdo Argentino-Brasileño de Biotecnología (CABIO) establecido en 1986. Distintos estudios plantean precisamente la conveniencia de dar prioridad a los esfuerzos tecnológicos de los países periféricos en la biotecnología y la química fina, ya que abarcan un amplio campo y podrían aplicarse en plazos relativamente cortos. Los éxitos del CABIO fueron limitados, pero esto no significa que deban abandonarse las estrategias de promoción de acuerdos tecnológicos a nivel zonal. Este estudio señala el diferente comportamiento en materia de dinamismo comercial y empresarial exhibido por aquellas empresas que realizaron innovaciones tecnológicas propias.

iii) La reducción de los obstáculos a la competencia que implican los mercados cautivos por las marcas, por medio de una más rápida transición hacia el consumo de productos genéricos (Katz, 1997). Este proceso está mostrando un mayor avance en Brasil. A nivel de las economías nacionales permitirá adelantar en la desregulación del mercado de medicamentos, minimizando el impacto negativo de ella sobre el bienestar del consumidor. En el plano zonal, acrecentará la capacidad de los laboratorios nacionales para competir en sus mercados. Uno de los problemas más serios que se presentan en este terreno es la falta de información de los médicos y consumidores respecto a la calidad de los medicamentos genéricos. La coordinación entre los países de la zona puede contribuir a aumentar su capacidad tanto en lo relacionado con las experiencias y leyes que faciliten esta transición como en todo aquello vinculado con la certificación de productos bioequivalentes. 
Bibliografía

Challú, P. y P. Lewis (1996): Adecuación de la ley argentina de patentes al GATT, Baltimore, Pennsylvania, Abeledo Perrot.

De Queiroz, S. (1993): Competitividade da indústria de fármacos, Estudo da competitividade da indústria brasileira, Campinas, Instituto de Economía, Universidad Estadual de Campinas (UNICAMP)/Instituto de Economía Industrial, Universidad Federal de Río de Janeiro (UFRJ)/ Food Drug Administration (FDA)/ Fundación Centro de Estudios de Comercio Exterior.

Katz, J. (1997): Apertura económica y desregulación en el mercado de medicamentos, Baltimore, Pennsylvania, Alianza Editorial.

Laens, S., F. Lorenzo y R. Osimani (1993): Macroeconomic Conditions and Trade Liberalization: The Case of Uruguay, Montevideo, CINVE.
Laens, S. y M. I. Terra (1998): Formación de la Unión Aduanera, sectores afectados y análisis de los flujos de comercio, Montevideo, mimeo.

Ministerio de Economía de la Provincia de Buenos Aires (1997): La industria farmacéutica argentina ante el nuevo contexto macroeconómico, 1991-1996, Cuaderno de economía de la Provincia de Buenos Aires, No 28, Buenos Aires.

Torrello, M y N. Noya (1992): Las políticas de incentivos a la inversión privada, Informe de investigación, $\mathrm{N}^{\circ} 23$, Montevideo, CINVE.

Watal, J. y A. P. Mathai (1995): Sectoral Impact of the Uruguay Round Agreements on Developing Countries: Pharmaceutical Industry, Nueva Delhi, Organización de las Naciones Unidas para el Desarrollo Industrial (ONUDI). 